\title{
2D Single Mode Optical Fiber Wave Guide Design for Multi Haul Applications
}

\section{Dr. Venkata Raghavendra Miriampally}

Associate Professor

Electronic \& Communication Program

School of Electrical Engineering \& Computing

Adama Science \& Technology University

Adama, Ethiopia

\author{
Mr. Tadesse Hailu
}

Program Chair

Electronic \& Communication Program

School of Electrical Engineering \& Computing

Adama Science \& Technology University

Adama, Ethiopia

\begin{abstract}
The optical communications had an exponential growth over the last few years, having a significant commercial market in optical systems and components. This growth has been extended across all application areas, from transcontinental and transoceanic distances to regional networks and lastly to campus and building wiring. This paper basically explains the concept of Optical fiber communication, the propagation of light inside the fiber and mainly the two dimensional design of optical fiber itself. The researcher discuss the parameters such as effective group delay, dispersion. Modes, Bending Loss, Material Loss, Mode field, Birefringence, Splice Loss, Polarization Mode Dispersion (PMD), Effect of nonlinear Refractive Index. Finally we design the optical fiber with all the above mentioned parameters.
\end{abstract}

Keywords: Group delay, Dispersion. Modes, Material Loss, Bending Loss, Splice Loss, Birefringence, Mode field, Polarization Mode Dispersion (PMD)

\section{INTRODUCTION}

In the early stages of development of technology, fiber communication promised extremely high data rates, which allow large amount of data to be transmitted at a higher data rate. It also had the potential for transmission over long distances without a repeater. The bandwidth of the fiber optic communication system, determines the maximum data rate and the major components of the system. Fig. 1 shows the block diagram of fiber optic communication system. The information signal that is to be transmitted may be computer data, video or voice. The first step is to convert the information into a form well suited with the communications medium. This is usually done by altering continuous analog signals such as TV (voice and video) signals into a series of digital pulses.

An Analog to digital converter is used for this purpose. These digital pulses are then used to flash a powerful light source to off and on very rapidly. In a simple system for short distances, the light source is usually a light emitting diode. This is a semiconductor device that gives low intensity red light. Other colors are also used. Another commonly used light source is the solid state laser that generates an extremely high intense coherent light beam. This light beam pulses are then fed into a fiber cable where they are communicated over long distances. At the receiving end, a light sensitive device known as a light detector or photocell is used to detect the light pulses. This photo detector or photocell converts the light pulses into an electrical signal.

The electrical pulses are then amplified and reshaped back into digital form. Both the sources at the sending end and the detectors on the receiving end must operate at the same data rate. The device that drives the light source and the circuit that amplify and process the detected light must have suitable high-frequency response. The fiber itself must not garble the high-speed light pulses used in the data transmission. They are given as an input to a decoder, such as a D/A, where the original data is recovered. In very long transmission systems, repeaters are used along the way. Since the light is significantly attenuated when it travels over long distances, at some distance it may be too weak to be received constantly. To overcome this difficulty, special repeater stations are used to pick up light beam, they convert it back to electrical pulses that are amplified and then re-transmit them. Several stages of repeaters are desired over very long distances. This attenuation is due to so many parameters and we will discuss them in detail as follows. 


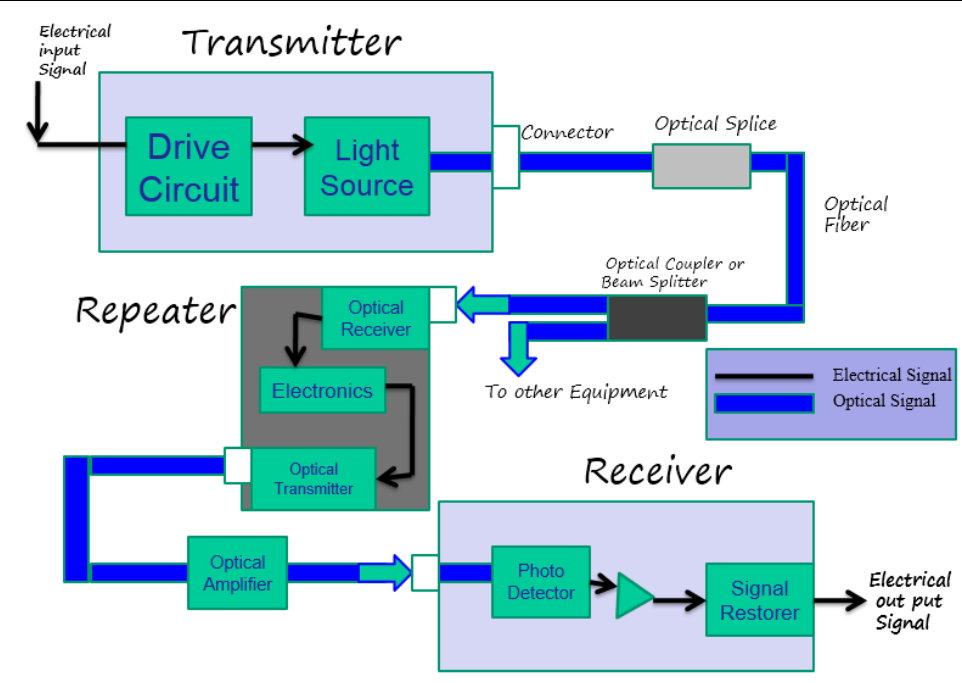

Fig1. Block diagram of Optical fiber Communication

\section{Materials ANd Methods}

The study and simulation has been performed with the help of Optifiber software by considering a single step index optical fiber with two different regions for two materials, one for core and the other for cladding having the index of refraction as 1.45213 and 1.44692 respectively. The simulation is conducted depending on different mathematical models of the parameters of optical fiber as follows.

\subsection{Effective Group Delay}

If the index of refraction of the fiber material varies with wavelength causing the group velocity to vary, it is represented as material dispersion.

The group delay $\boldsymbol{T}_{\boldsymbol{g}}$ is given by the product of the transmission distance $\mathrm{z}$ by the first derivative of frequency of the propagation constant:

$T_{g=z \frac{d \beta}{d \omega}=z \frac{d \lambda d\left(n k_{0}\right)}{d \omega} d_{\lambda}} ;$ Where $\mathrm{n}$ is the refractive index; $\lambda=\frac{2 \pi c}{\omega}, k_{0}=\frac{2 \pi}{\lambda} ;$

We have $\boldsymbol{T}_{\boldsymbol{g}}=\frac{-2 \pi \boldsymbol{c} \boldsymbol{z}}{\boldsymbol{\omega}^{2}}\left(k_{o} \frac{\boldsymbol{d} \boldsymbol{n}}{\boldsymbol{d}_{\lambda}}+\frac{\boldsymbol{d} \boldsymbol{k}_{\mathbf{0}}}{\boldsymbol{d}_{\lambda}}\right)=\frac{z}{\boldsymbol{c}}\left(n-\lambda \frac{d n}{d \lambda}\right)$

The dispersion coefficient $\mathrm{D}$ is defined as $D=\frac{d T_{g}}{d \lambda}$; in bulk materials we have $D=-\frac{z}{c} \lambda \frac{d^{2} n}{d \lambda^{2}}$; The Group delay Vs. Wavelength is shown in Fig. 5.

\subsection{Material dispersion of the fiber}

We have an equation that the velocity of light in a medium is given by $\mathrm{v}=c / n$. where $n$ is the refractive index of the medium, which depends on the wavelength. This dependence of the refractive index on wavelength leads to dispersion, if a white pencil light beam incident on a prism. Since the refractive index of glass depends on the wavelength, the angle of refraction will be different for different colors. The incident light will therefore disperse into its constituent colors, the dispersion will become more evident at the second surface of the prism. The quantity $v$ defined is usually referred to as the phase velocity.

However, a pulse travels with is known as the group velocity, which is given by $\mathrm{v}_{\mathrm{g}}=c / n_{\mathrm{g}}$; where $n_{\mathrm{g}}$ is known as the group index and, in most cases its value is slightly larger than $n$. In Table 1 and in Fig. 2 we have represented $n$ and $n_{\mathrm{g}}$ for pure silica for varying wavelength between $700 \mathrm{~nm}$ and $1600 \mathrm{~nm}$.

Table1: $n$ and $n_{g}$ for pure silica for varying wavelength between $700 \mathrm{~nm}$ and $1600 \mathrm{~nm}$.

\begin{tabular}{|l|l|l|l|}
\hline$\lambda 0(\mathrm{~nm})$ & $n(\lambda 0)$ & $n_{\mathrm{g}}(\lambda 0)$ & $D m(\mathrm{ps} / \mathrm{nm}-\mathrm{km})$ \\
\hline 700 & 1.45561 & 1.47154 & -172.902 \\
\hline 750 & 1.45456 & 1.46924 & -135.313 \\
\hline 800 & 1.45364 & 1.46744 & -106.609 \\
\hline 850 & 1.45282 & 1.46601 & -84.2077 \\
\hline 900 & 1.45208 & 1.46489 & -66.382 \\
\hline
\end{tabular}


2D Single Mode Optical Fiber Wave Guide Design for Multi Haul Applications

\begin{tabular}{|l|l|l|l|}
\hline \hline 950 & 1.45139 & 1.46401 & -51.9441 \\
\hline 1000 & 1.45075 & 1.46332 & -40.0577 \\
\hline 1050 & 1.45013 & 1.46279 & -30.1214 \\
\hline 1100 & 1.44954 & 1.46241 & -21.6951 \\
\hline 1150 & 1.44896 & 1.46214 & -14.4511 \\
\hline 1200 & 1.44839 & 1.46197 & -8.14213 \\
\hline 1250 & 1.44783 & 1.46189 & -2.57872 \\
\hline 1300 & 1.44726 & 1.46189 & 2.38579 \\
\hline 1350 & 1.44670 & 1.46196 & 6.86631 \\
\hline 1400 & 1.44613 & 1.46209 & 10.9539 \\
\hline 1450 & 1.44556 & 1.46229 & 14.7211 \\
\hline 1500 & 1.44498 & 1.46253 & 18.2268 \\
\hline 1550 & 1.44439 & 1.46283 & 21.5187 \\
\hline 1600 & 1.44379 & 1.46318 & 24.6358 \\
\hline
\end{tabular}

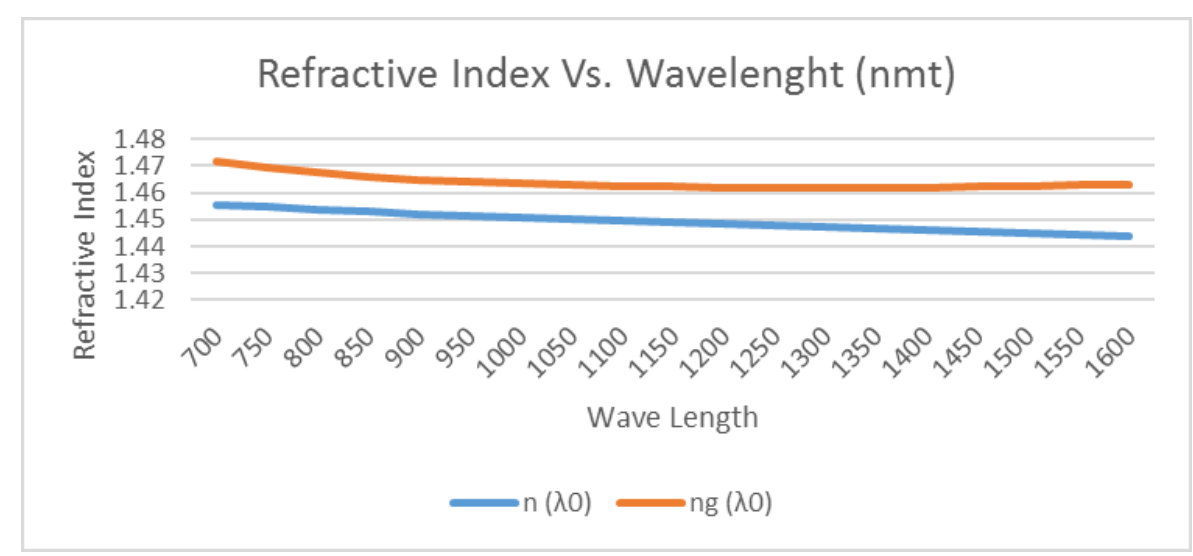

Fig2. Refractive Index vs. Wavelength (nmt)

In a fiber, the core and cladding are fabricated with different materials. We assume that there are $\mathrm{L}$ layers in the fiber cross-section, each layer has its own refractive index. The refractive index profile of simulated output is shown in Fig. 3 and modal refractive index vs. wavelength is represented in Fig. 4.

The total material dispersion of the optical fiber is calculated by Eq.1as below:

$D(\lambda)=-\frac{z}{c} \sum_{i=1}^{L} \Gamma_{i} \frac{d^{2} n_{i}}{d \lambda^{2}}$

Where $\Gamma_{i}$ is the confinement factor of each layer. The confinement factor is the portion of total power guided in the $\mathrm{i}^{\text {th }}$ layer.

\subsection{Waveguide dispersion of the fiber}

Wavelength dependence of the effective refractive index $N_{\text {eff }}$ of the fiber mode results in Waveguide dispersion. The waveguide dispersion is calculated by:

$$
D_{w g}=-\frac{z}{c} \lambda \frac{d^{2} N_{e f f}}{d \lambda^{2}}
$$

\subsection{Total dispersion of the fiber}

The total dispersion is the total effect of different dispersions such as material and waveguide. First the material dispersion effect will be calculated. Then the mode solver calculates the mode effective index $N_{\text {eff }}$. The total dispersion of a fiber is expressed in Eq.2:

$D_{\text {tot }}=-\frac{z}{c} \lambda \frac{d^{2} N_{e f f}}{d \lambda^{2}}$

The total dispersion vs. wavelength is shown in Fig. 6 .

\subsection{Mode Field Diameter and Mode Area Definitions}

\section{Mode field diameter and area importance}

The Mode Field Diameter is an important parameter related to the optical field distribution in the fiber. It has been shown that MFD provides useful evidence about the fiber cabling behavior, such as possible joint, macro bending, and micro bending losses. The actual area of the fibers has a relation to the nonlinear distortions in long fiber links. 


\section{Near-field diameter definition}

The near-field Mode Field Diameter is also known as the "Petermann I" diameter. It is defined as the diameter at which the near field power falls to $\frac{1}{e^{2}}$ of its maximum value. It can be calculated by Eq.3.

$$
[1]: d_{n}=2 \sqrt{2}\left(\frac{\int_{0}^{\infty} E^{2}(r) r^{3} d r}{\int_{0}^{\infty} E^{2}(r) r d r}\right)^{1 / 2}
$$

Where $\mathrm{E}(\mathrm{r})$ is optical mode field distribution.

\section{Far-field diameter definition}

The far-field Mode Field Diameter is also known as the "Petermann II" diameter. It is defined as the diameter at which the far field power falls to $\frac{1}{e^{2}}$ of its maximum value. It can be calculated by Eq.4.

$[1]: d_{n}=2 \sqrt{2}\left(\frac{\int_{0}^{\infty} E^{2}(r) r d r}{\int_{0}^{\infty}\left[E^{\prime}(r)\right]^{2} r d r}\right)^{1 / 2}$

Where $\mathrm{E}(\mathrm{r})$ is the optical mode field distribution, and prime denotes the first derivative of $\mathrm{E}(\mathrm{r})$

\section{Effective mode area definition}

The effective Mode Area is calculated by Eq.5 as

$A_{e f f}=\frac{\left[\int_{-\infty}^{\infty} \int_{-\infty}^{\infty}|E(x, y)|^{2} d_{x} d_{y}\right]^{2}}{\int_{-\infty}^{\infty} \int_{-\infty}^{\infty}|E(x, y)|^{4} d_{x} d_{y}}$

Where $\mathrm{E}(\mathrm{x}, \mathrm{y})$ is the optical mode field distribution

\section{Effective mode field diameter definition}

Effective Mode Field Diameter defined in Eq.6 as:

$d_{e f f}=\frac{2 \sqrt{2} \int E_{i}{ }^{2} r d r}{\left[\int E_{i}{ }^{4} r d r\right]^{\frac{1}{2}}} \Rightarrow>d_{e f f}=\frac{2}{\sqrt{\pi}} \sqrt{A_{e f f}}$

Where $\mathrm{E}(\mathrm{r})$ is the optical mode field distribution. The simulation result for Mode field diameter vs. wavelength is shown in Fig. 7. The confinement of the core and cladding can be observed from Fig. 11 and the practical MFD is shown in Fig. 12.

\subsection{Fiber Loss Models}

\section{Fiber propagation loss definition}

The total fiber loss can be divided into fiber induced losses and material losses. Material losses include Rayleigh scattering, ultraviolet, infrared absorption, and hydroxyl absorption losses. Material losses are the restraining losses in fibers. Fiber loss is defined as the ratio of the output optical power $P_{\text {out }}$ from a fiber of length $L$ to the input optical power $P_{\text {in }}$. The symbol $\alpha$ is commonly used to express loss in $\frac{d B}{K m}$ and is expressed in Eq.7 as

$\propto=\frac{10}{L} \log \left(\frac{P_{\text {in }}}{P_{\text {out }}}\right)$.

And the material loss vs. wavelength is depicted in Fig. 8.

\section{Rayleigh scattering model}

Light transmitted through the fiber suffers scattering loss due to rough appearance of atoms or molecules of the glass fiber, which is known as Rayleigh scattering loss. The fiber loss is expressed in $\frac{d B}{K m}$ through [2]: $\propto_{s}=\frac{A}{\lambda^{2}}$; for a single-component glass such as $\operatorname{Sio}_{2} A=\frac{8 \pi^{3}}{3} n_{0} P^{2} \beta k T$; Where $n_{0}$ is the refractive index, $\mathrm{p}$ is the photoelastic coefficient, $\beta$ is the thermal compressibility, $\mathrm{k}$ is the Boltzmann coefficient, and $\mathrm{T}$ is the absolute temperature of the sample.

\section{Macro bending loss model}

The macro bending loss is a radiative loss occurs when the fiber bend radius is large compared to the fiber diameter. It is defined as usual by $P(Z)=P(0) e^{-\gamma z}$; where $P(0)$ the input is power and $P(Z)$ is the output power at distance $\mathrm{z}$ respectively. 
There are two models for Macro bending. The first uses the closed-form integral formula [3]. Using this the macro bending power loss coefficient is expressed as a function of the bending radius $R_{b}$ in the form of Eq.8:

$\gamma=\frac{\sqrt{\pi}\left(\frac{P_{c l a d}}{P}\right)}{2 s r_{c}\left[K_{v-1}(W) K_{v+1}(W)-K_{v}{ }^{2}(W)\right]} \frac{\exp \left(\frac{-4 \Delta W^{3}}{3 r_{c} V^{2}} R_{b}\right)}{W\left(\frac{W R_{b}}{r_{c}}+\frac{v^{2}}{2 \Delta W}\right)^{\frac{1}{2}}}$

The parameters appearing above are given by:

$V=K_{0} r_{c} \sqrt{N_{\max }^{2}-} N_{c l a d}^{2}$ is the normalized dimensionless frequency;

$W=r_{c} \sqrt{\beta^{2}-\left(k_{0} N_{\text {clad }}\right)^{2}} ; \Delta=\frac{\left(N_{\max }{ }^{2}-N_{\text {clad }}{ }^{2}\right)}{2 N_{\max }{ }^{2}} ;$ where $r_{c}$ designates the fiber core radius, $N_{\max }$ is the maximum refractive index and $N_{\text {clad }}$ is the cladding refractive index, $\beta$ is the mode propagation constant, $K_{0}$ is the propagation constant in vacuum, $v$ is the azimuthal mode number, $s=2$ if $V=0$ or $s=1$ if $V \neq 0$ and $K_{v}$ is the modified Bessel function of the second kind of order $v$.

Second macro bending loss model is expressed as Eq.9 [4]:

$\gamma=\left(\frac{\pi V^{8}}{16 r_{c} R_{b} W^{3}}\right)^{1 / 2} \exp \left(\frac{-4 \Delta W^{3} R_{b}}{3 r_{c} V^{2}}\right) \frac{\left[\int_{0}^{-\infty}(1-f) F_{0} R d R\right]^{2}}{\int_{0}^{\infty} F_{0}^{2} R d R}$

Where $F_{0}$ is the radial field of the fundamental mode.

$f=\frac{N_{\max }{ }^{2}-N(R)^{2}}{N_{\max }{ }^{2}-N_{\text {clad }}}$; and $\mathrm{N}(\mathrm{R})$ is the refractive index profile of the fiber. The other parameters are given above. The two models give similar results for step-index fibers. The loss coefficient $\gamma$ can be converted to loss in $\frac{d B}{K m}$ units as follows:

$$
\propto_{\text {macro }}=\frac{10}{L} \log \left(\frac{P_{\text {in }}}{P_{\text {out }}}\right)=\frac{10}{L} \log [\exp (\gamma L)]=\frac{10}{\ln (10)} \cdot \gamma
$$

\section{Micro bending loss model}

Micro bending loss is also a radiative loss in fiber resulting from mode coupling caused by random micro bends, which are repetitive small fluctuations in the radius of the curvature of the fiber axis. An approximate expression for the attenuation coefficient is given by [5]:

$\left.\propto_{\text {micro }}=A\left(k n_{1} d_{n}\right)^{2}\right)\left(k n_{1} d_{n}{ }^{2}\right)^{2 p}$; Where $A$ is a constant, $d_{n}$ is the near field diameter $n_{1}$ is the core refractive index, $\mathrm{k}$ is the free space wave number, and $\mathrm{p}$ is the exponent in the power law. The simulation result for total bending loss vs. wavelength is shown in Fig. 9.

\section{Splice loss model}

A splice is a joint (dielectric interface between two optical fibers). Any refractive index mismatch will produce reflection and refraction at any point in this interface. For splicing calculations, we assume that the mode field of single-mode fiber is nearly Gaussian. The coupling losses for the splice can be calculated by evaluating the connection between two misaligned Gaussian beams. Based on the above model, the coupling loss between two single mode fibers is given by Eq.11 [6]:

$\propto_{\text {splice }}=-10 \log \left[\left(\frac{16 n_{1}{ }^{2} n_{2}{ }^{2}}{\left(n_{1}+n_{2}\right)^{2}}\right) \frac{\sigma}{q} \exp \left(\frac{-\rho u}{q}\right)\right]$

Where $\rho=\frac{\left(k w_{1}\right)^{2}}{2} ; q=G^{2}+\frac{(\sigma+1)^{2}}{4}$

$u=(\sigma+1) F^{2}+2 \sigma F G \sin \theta+\sigma\left(G^{2}+\frac{\sigma+1}{4}\right) \sin ^{2} \theta ; F=\frac{x}{k w_{1}^{2}} ; G=\frac{z}{k w_{1}^{2}} ; \sigma=\left(\frac{w_{2}}{w_{1}}\right)^{2} ; k=\frac{2 \pi n_{2}}{\lambda}$

Where $n_{1}$ is Core refractive index of the fiber, $n_{2}$ is Refractive index of the medium between the two fibers, $\lambda$ is Wavelength, $w_{1}$ is Near field mode field radius of transmitting fiber, $w_{2}$ is Near field mode field radius of receiving fiber, $x$ is Lateral offset, $z$ is Longitudinal offset, $\theta$ is Angular misalignment. The simulation result for total Splice loss vs. wavelength is shown in Fig. 10. 


\subsection{Fiber Birefringence Models}

\section{Fiber birefringence definition}

The difference between the propagation constants of the polarization Eigen modes is defined as fiber birefringence, that is: $\Delta \beta=\beta_{x}-\beta_{y}$

The Differential Group Delay per unit length is defined by Eq.12:

$\partial \tau=\frac{\partial \Delta \beta}{\partial \omega}=-\frac{\lambda^{2}}{2 \pi c} \frac{\partial \Delta \beta}{\partial \lambda}$

\section{Intrinsic perturbations birefringence}

Intrinsic perturbations are generally have a huge impact in step index fibers only and they are exist during the manufacturing process and are permanent feature of the fiber. They include a noncircular core and nonsymmetrical stress fields in the glass around the core region. A noncircular core results in geometric birefringence, whereas a nonsymmetrical stress field results in stress birefringence.

\section{Elliptical core birefringence definition}

The geometrical variation of a non-circular core introduces a linear birefringence in the optical fiber. This birefringence depends strongly on normalized frequency $\mathrm{V}$, at which the fiber is being operated. The ellipticity (non circularity) of the core is defined by the parameter $\varepsilon$ and $\varepsilon=1-\frac{a}{b}$ Where a, b are the minor and major axis of the elliptical core respectively. The birefringence induced by an elliptical core is linear.

In the case of a step index fiber, the birefringence $\Delta \beta_{\text {ell }}=\beta_{x}-\beta_{y}$ is given by Eq.13 [7]:

$$
\Delta \beta_{\text {ell }}=\varepsilon n_{1} k_{0} \Delta^{2} G(V)
$$

Where $\Delta=\frac{n_{1}-n_{2}}{n_{1}}$ is the refractive index difference between the core and the cladding.

The wave propagation constant is $k_{0}=\frac{2 \pi}{\lambda}$; the function $G(V)$ in the birefringence formula is given in Eq.14 as

$G(V)=\frac{W^{2}}{V^{4}}\left[U^{2}+\left(U^{2}+W^{2}\right)\left[\frac{J_{0}(U)}{J_{1}(U)}\right]^{2}+U W^{2}\left[\frac{J_{0}(U)}{J_{1}(U)}\right]^{3}\right]$

With the following definitions of waveguide parameters as shown from Eq.15 to Eq.18

$U=a \sqrt{n_{1}{ }^{2} k_{0}{ }^{2}-\beta^{2}}$

$W=a \sqrt{\beta^{2}-n_{2}{ }^{2} k_{0}{ }^{2}}$

$V=\sqrt{U^{2}+W^{2}}=a k_{0} \sqrt{n_{1}^{2}-n_{2}^{2}}$

Where a is the core radius.

The Differential Group Delay per unit length is $\partial \tau_{e l l}=\frac{\varepsilon n_{1} \Delta^{2}}{c} \frac{d}{d V}[V G(V)]$

Where $\mathrm{c}$ is the speed of light in vacuum.

\section{Extrinsic perturbations birefringence}

Birefringence can also be formed in a fiber when it is imperiled to external forces in handling or cabling. Such extrinsic sources of birefringence comprise of lateral stress, fiber bending and fiber twisting. All three of these mechanisms are usually present to some extent in coiled and field-installed telecommunications fiber.

\section{Lateral stress birefringence definition}

When two forces of equal and opposite in magnitude 'p' act across an axis of a fiber with a diameter $\mathrm{d}$, the birefringence persuaded is linear. The firm axis of birefringence is aligned with the axis represented by the two opposite forces. The birefringence is given by Eq.19 as [7]: 
$\Delta \beta_{\text {lateral stress }}=-8 \frac{C_{p} k_{0}}{\pi d}\left[1-\left(\frac{a}{d}\right)^{2} H(V)\right]$

Where ' $\mathrm{C}$ ' is Photoelastic constant, ' $\mathrm{p}$ ' is Lateral force, $k_{0}$ is Wave propagation constant in vacuum, $\mathrm{d}$ is the outer diameter of the fiber, ' $\mathrm{a}$ ' is the average core radius. Internal stress birefringence definition. The Differential Group Delay per unit length $\partial \tau_{\text {lateral stress }}$ is expressed in Eq.20:

$\partial \tau_{\text {lateral stress }}=-8 \frac{C_{p} k_{0}}{\pi c d}\left[1-\left(\frac{a}{d}\right)^{2} H(V)+V \frac{d H(V)}{d V}\right]$

\section{Bending birefringence definition}

Linear birefringence is a result of bending on a fiber with a bending radius $\mathrm{R} \gg>$ a (fiber core). The firm axis of birefringence is at a right angle to the bending plane.

The slow axis is aligned with the bending radius. The birefringence is given by Eq.21 [7]:

$\Delta \beta_{\text {Bending }}=-\frac{1}{8}\left(\frac{d}{R}\right)^{2} \frac{E C k_{0}}{\pi d}\left[1-\frac{1}{3}\left(\frac{a}{d}\right)^{2} H(V)\right]$

With ' $R$ ' being the bending radius, and ' $d$ ' is The outer diameter of the fiber, ' $E$ ' is The Young modulus, ' $\mathrm{C}$ ' is Photo elastic constant, ' $k_{0}$ ' is Wave propagation constant in vacuum, ' $\mathrm{a}$ ' is the average core radius and, The Differential Group Delay per unit length is expressed as Eq.22:

$\partial \tau_{\text {Bending }}==-\frac{E C}{8 c}\left(\frac{d}{R}\right)^{2}\left[1-\frac{1}{3}\left(\frac{a}{d}\right)^{2}\left[H(V)+V \frac{d H(V)}{d V}\right]\right]$

The simulation result for Birefringence vs. wavelength is shown in Fig. 13.

\subsection{Polarization Mode Dispersion Models}

\section{Polarization mode dispersion}

Propagation constants of the two polarization Eigen modes that are degenerate in ideal single-mode fibers, in real telecommunications fibers, perturbations performance on the fiber in a way that it induces a birefringence. Consequently, when a pulse is made to transmit through a fiber, it results in a differential group delay between the two polarization Eigen modes. The stochastic behavior of these perturbations results in a phenomenon of random mode coupling which makes impossible about differential group delay.

\section{Principal states of polarization}

The Principal States of Polarization model [8] is based on the observation that at any given optical frequency, there occurs a set of two mutually perpendicular input principle states of polarization for which the corresponding output states of polarization are independent of frequency. The Differential Group Delay resulting from Polarization Mode Dispersion is then defined between the two output Principal States of Polarizations.

The birefringence in telecommunication single-mode fibers varies arbitrarily along the fiber length, a product of variation in the drawing and cabling process. Furthermore, due to the temperature dependence of many of the fluctuations that present in the fiber during manufacturing, the transmission properties typically change as ambient temperature changes. In practice, these perturbations in temperature strongly affect Polarization mode dispersion time evolution. To assess properties of long fiber distances, one can adopt a statistical approach. In this case of long distance fibers, the polarization Eigen states can only be defined nearby and the birefringence vector has to be stochastic. The simulation result for polarization mode dispersion vs. wavelength is shown in Fig. 14.

\section{Dispersion vector}

In the time domain, the Polarization Mode Dispersion induces a time shift between the two Principal States of Polarization. In the frequency domain, the output PSPs undergoes a rotation on the Point care sphere about an axis connecting the two PSPs.

The rate and direction of rotation is given by the dispersion vector $\Omega(\omega, z)$ in Eq. 23 given by: $\Omega(\omega, z)=\Delta \tau . P_{b}$

Where $P_{b}$ represents the negative output principal state. 
The strength of the dispersion vector $\Omega(\omega, z)$ is equal to the differential delay time $\Delta \tau$ between the two output principal states, where its combined Stokes vector corresponds to the Stokes vector of the negative output principal state. The direction of the Dispersion Vector $\Omega$ defines an axis whose two intercepts with the surface of the Poincare sphere correspond to the two principal states of polarization at the fiber output.

Where The Poincare sphere is a graphical tool that allows convenient description of polarized signals and polarization transformations during propagation. A point within a unit sphere can uniquely represent any state of polarization, where circular states of polarization are located at the poles. The coordinates of a point within or on the Poincare sphere are the normalized Stokes parameters.

\section{Effect of nonlinear Refractive Index}

Usually one of the design goals when constructing a fiber is to minimize its nonlinearities. The effective nonlinear coefficients of optical fibers depend on the nonlinear indices of the bulk materials building the fiber and on its wave guiding properties: shape of modes, degree of confinement, etc. As a result it can vary within broad limits. The effective nonlinear coefficient are represented as Eq.24 [9]:

$$
n_{2} e^{e f f}=\frac{\int_{-\infty}^{\infty} \int_{-\infty}^{\infty} n_{2}(x, y)|F(x, y)|^{4} d x d y}{\int_{-\infty}^{\infty} \int_{-\infty}^{\infty}|F(x, y)|^{2} d x d y}
$$

Where $n_{2}(x, y)$ is the user-defined spatially dependent nonlinear refractive index of the various fiber layers and $F(x, y)$ is the normalized mode field pattern. The simulation result for effective cladding refractive index vs. wavelength is shown in Fig. 15.

\section{Simulation \& Results}

The simulation is done having the view of the following parameters

Refractive Index Profile; Four regions are considered; Wave Length used is $1.3 \mu \mathrm{m}$.

\section{Region 0}

Starting Index: 1.45, Width $10 \mu \mathrm{m}$,

\section{Material Properties}

Host- pure Silica, Dopant + is 3.1\% germanium doped silicon,

Dopant - is $1 \%$ Fluorine doped silica

\section{Region 1}

Starting Index: 1, Width $20 \mu \mathrm{m}$,

The outputs for the simulation are as follows:

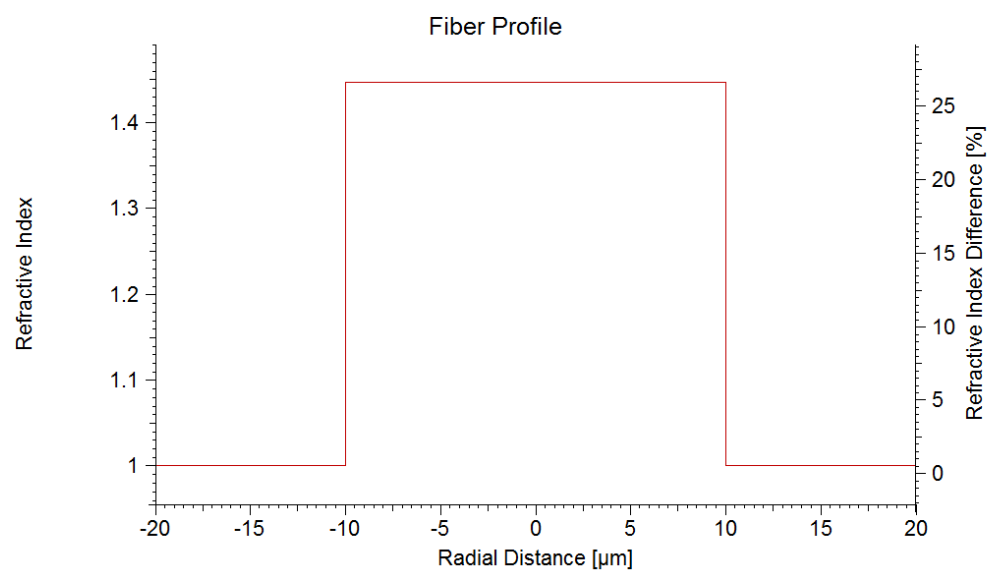

Fig3. Refractive Index Profile 


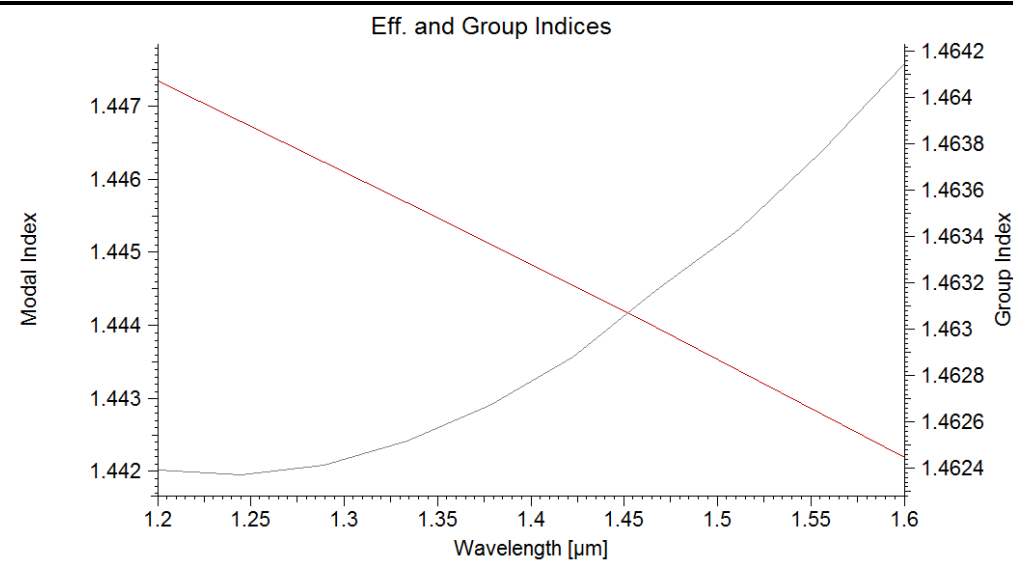

Fig4. Modal Index vs. Wavelength

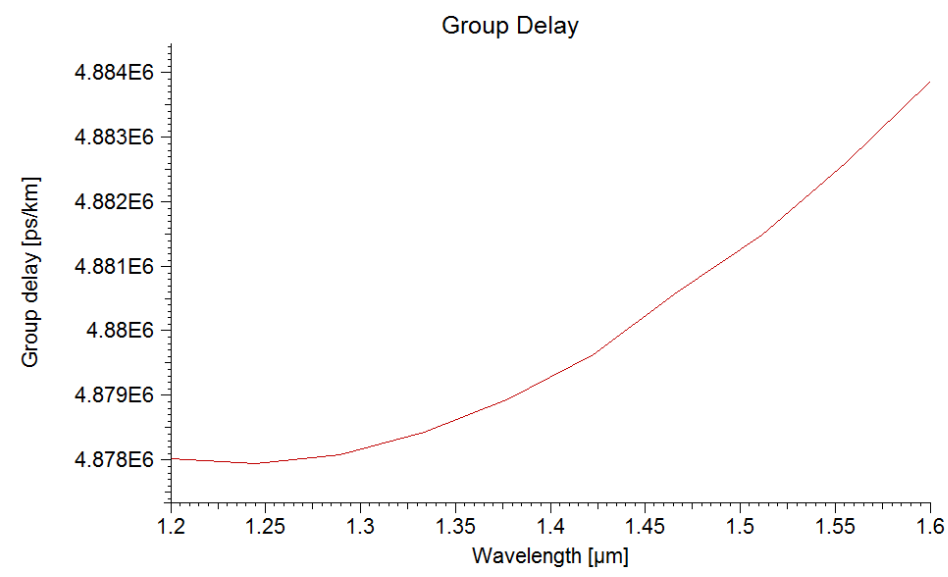

Fig5. Group delay vs. Wavelength

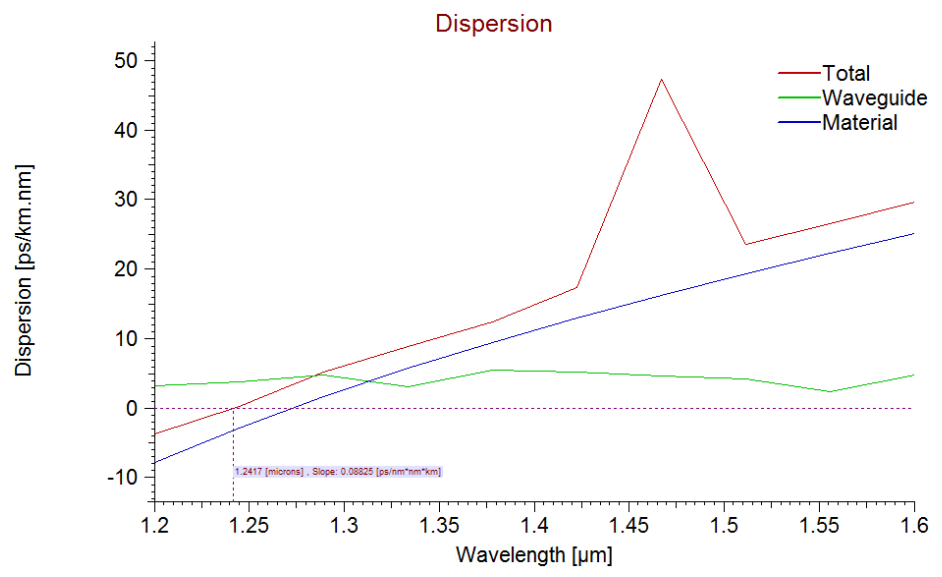

Fig6. Dispersion vs. Wavelength

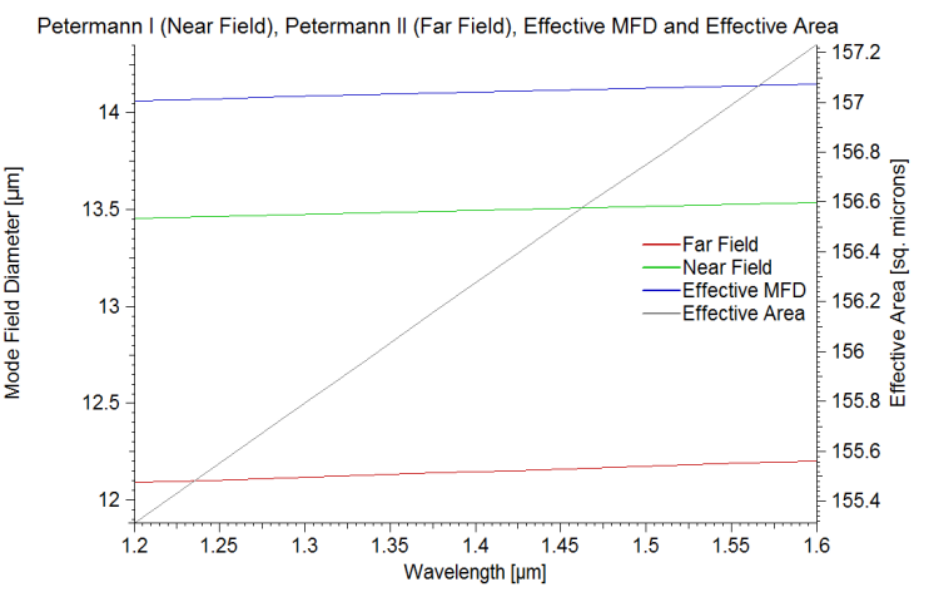

Fig7. Mode Field vs. Wavelength 
Dr. Venkata Raghavendra Miriampally \& Mr. Tadesse Hailu

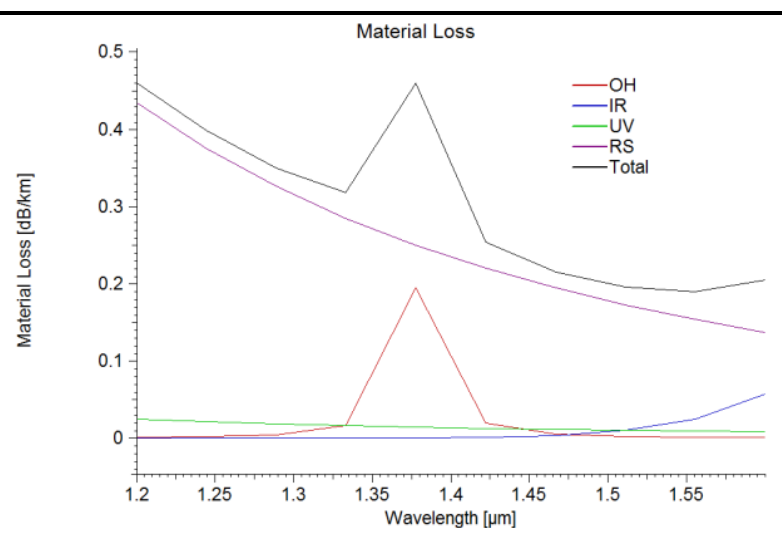

Fig8. Material Loss vs. Wavelength

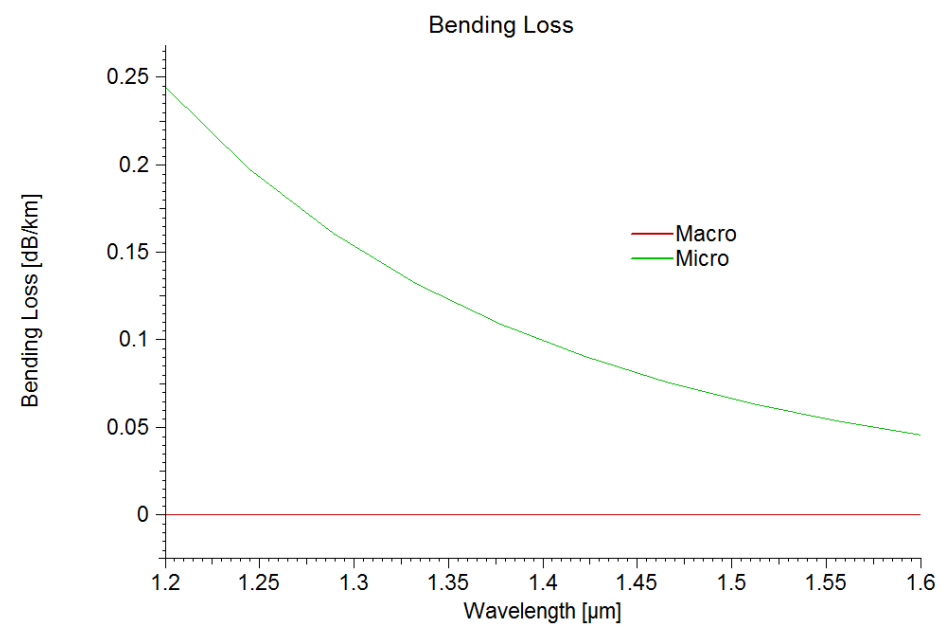

Fig9. Bending Loss vs. Wavelength

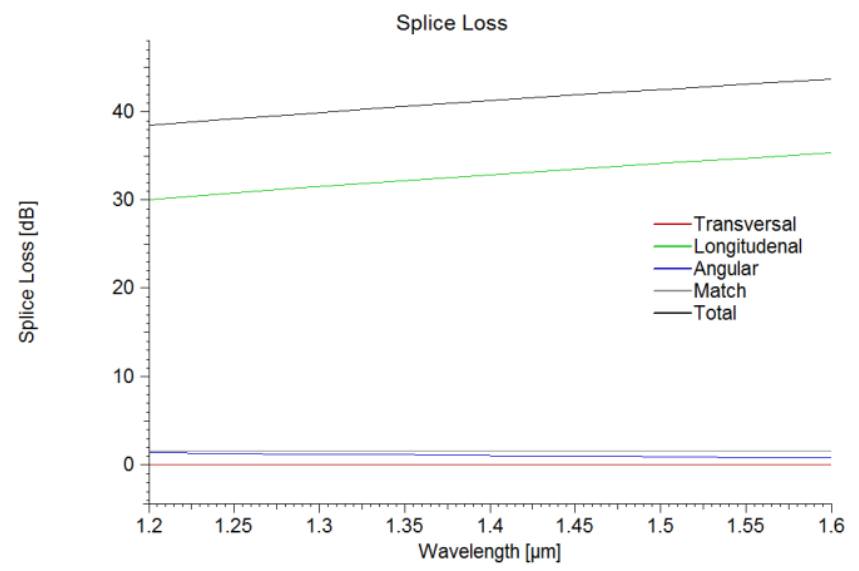

Fig10. Splice Loss vs. Wavelength

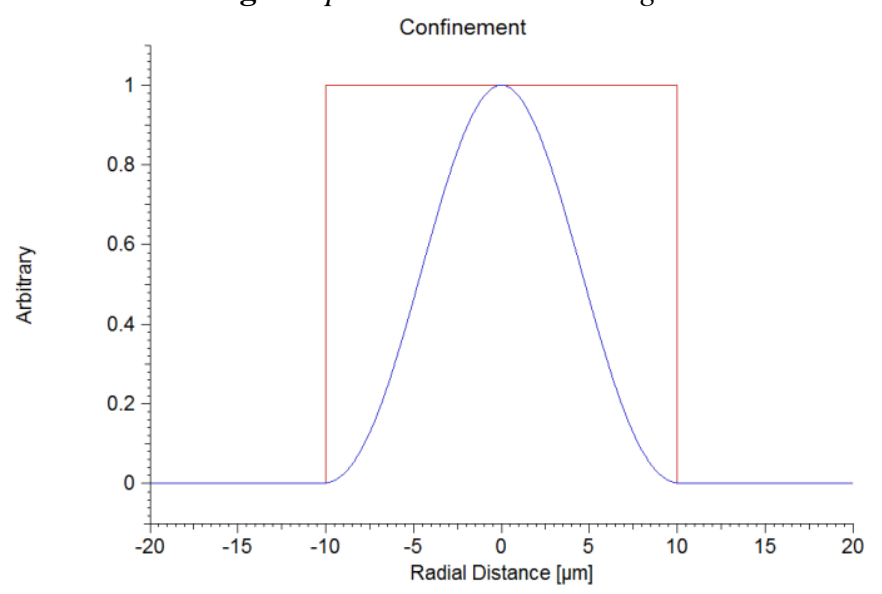

Fig11. Confinement 


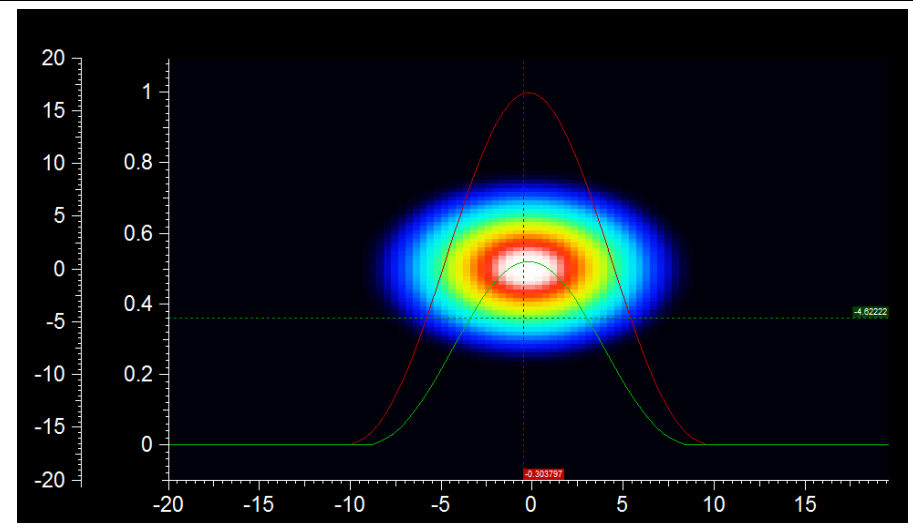

Fig12. Mode field Diameter.

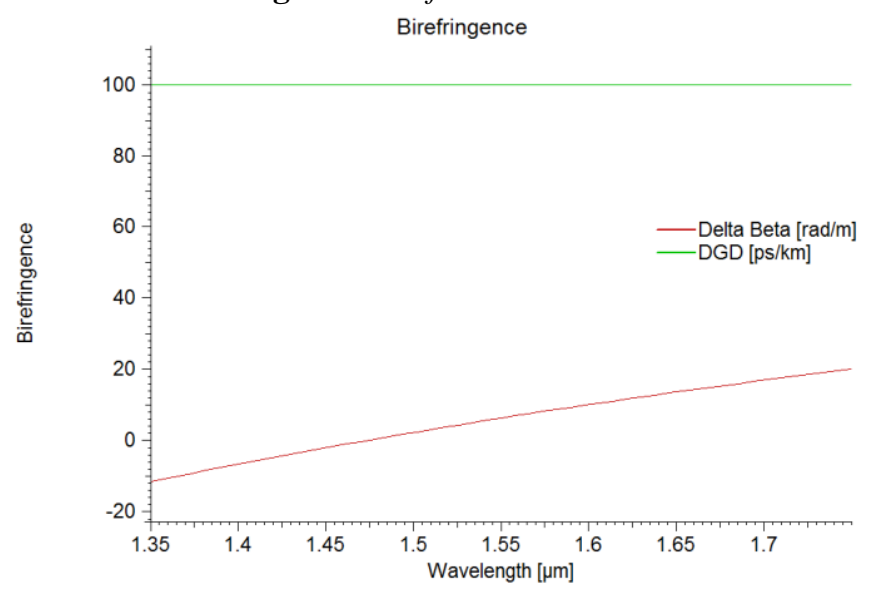

Fig13. Birefringence vs. Wavelength

Polarization mode dispersion

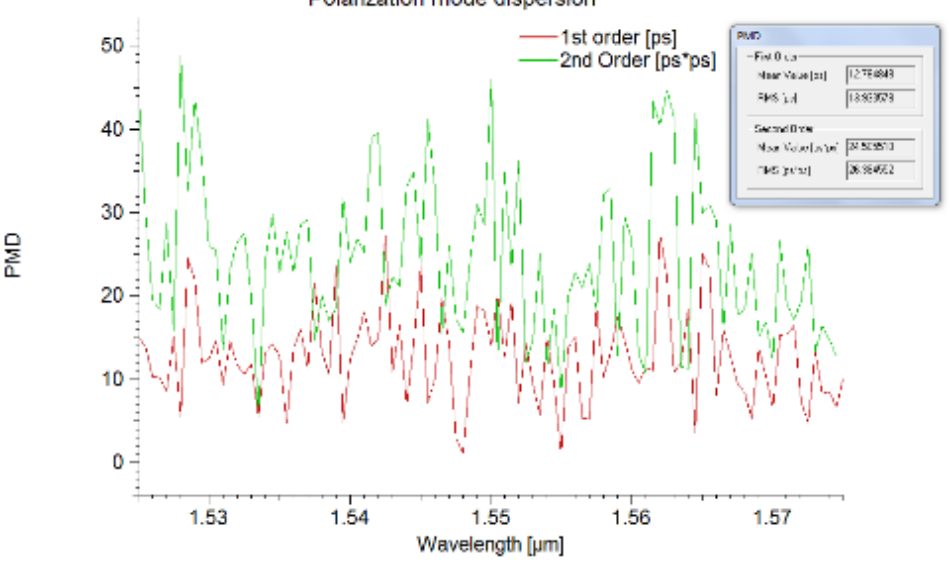

Fig14. Polarization Mode Dispersion vs. Wavelength

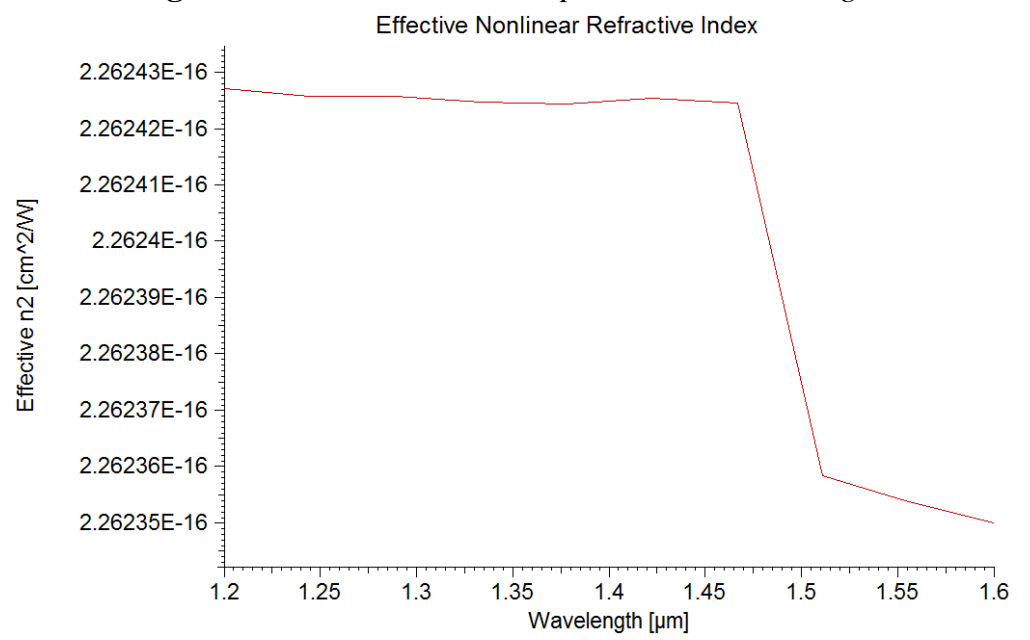

Fig15. Effective Cladding Refractive Index vs. Wavelength 


\section{Conclusion}

This paper has explained the concept of propagation of light with the two dimensional design of optical fiber. The parameters such as effective group delay, dispersion. Modes, Material Loss Bending Loss, Splice Loss, Mode field, Birefringence, Polarization Mode Dispersion (PMD), and Effect of nonlinear Refractive Index are clearly explained with their mathematical models and finally designed the optical fiber model with all the above mentioned parameters. The results are more accurate than the previous existing optical fiber technology.

\section{REFERENCES}

[1] M. Artiglia, "Mode field Diameter measurements in single-mode optical fibers," J. Lightwave Tech. Vol. 7, no. 8, pp. 11391152, 1989.

[2] J. A. Buck, "Fundamentals of Optical fibers," John Wiley \& Sons, 1995.

[3] J. Sakai, and T. Kimura, "Bending loss of propagation modes in arbitrary-index profile optical fibers", Applied Optics vol. 17, no. 10, pp 1499-1506, May 1978

[4] A. W. Snyder and J. D. Love, "Optical Waveguide Theory," Chapman and Hall, 1983.

[5] K. Petermann, "Microbending loss in monomode fibers," Electron. Lett., vol. 20, no. 3, pp. $107-$ 109, 1976.

[6] S. E. Miller and I. P. Kaminow, Eds., "Optical Fiber Telecommunications II," Academic Press, 1988.

[7] J. Sakai and T. Kimura, "Birefringence and Polarization Characteristics of Single-Mode optical Fibers under Elastic Deformations", IEEE Journal of Quantum Electronics, vol. QE-17, no. 6,

[8] C. D. Poole, N. S. Bergano, R. E. Wagner, and H. J. Schulte, "Polarization Dispersion and Principal States in a 147-km Undersea Lightwave Cable", Journal of Lightwave Technology, vol. LT-6, no. 7, pp 1185-1190, July 1988. pp 1041-1051, June 1981.

[9] D. Marcuse, “Theory of Dielectrical Optical Waveguides, Second Ed.,” Academic Press, 1991.

\section{AUTHORS' BIOGRAPHY}

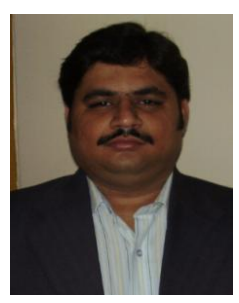

Dr. Venkata Raghavendra Miriampally is currently working as Associate Professor, Electronic and Communication Engineering Program, School of Electrical Engineering \& Computing, Adama Science \& Technology University, Ethiopia. His field of interest is Optical Communication. He has received his M.Tech from Electronics \& Communication Engineering. His main research includes signal estimation \& evaluation of optical communication, Satellite communication \& Microwaves. He has published papers in reputed national \& international Journals. He has participated in different national \& international conferences. He is a life member of ISTE, IE, ISOI, MIAENG, MIACSIT, and MAIRCC.

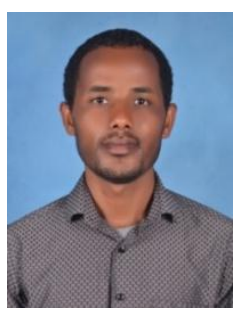

Mr.Tadesse Hailu Ayane received his B.Sc in Electrical Engineering degree from Jimma University in 2008 and Masters in Electronic and Computer Engineering degree from Addis Ababa University in 2010. He is a member of Ethiopian Society of Electrical Engineer since 2009. He worked in Midroc Gold Mine from 2008 - 2009 as Electrical Engineer. He has an experience of teaching in different Governmental universities with lecturer position since 2010. Currently, he is a lecturer and Program head of Electronic \& communication Engineering, Adama science and technology University, Ethiopia. His research interest includes IC and nanotechnology, signal processing, electronic automation, satellite technology. 